九州大学学術情報リポジトリ

Kyushu University Institutional Repository

\title{
ESTIMATION OF JUMP REGRESSION FUNCTION
}

Qiu, Peihua

Department of Statistics and Operations Research, Fudan University

Asano, Chooichiro

Research Institute of Fundamental Information Science, Kyushu University $\rightarrow$ Department of Information System Science, Soka University

\section{Li, Xianping}

Department of Statistics and Operations Research, Fudan University

https://doi.org/10.5109/13419

出版情報: Bulletin of informatics and cybernetics. 24 (3/4), pp.197-212, 1991-03. Research Association of Statistical Sciences

バージョン :

権利関係 : 


\title{
ESTIMATION OF JUMP REGRESSION FUNCTION
}

\author{
By
}

\author{
Peihua QIU*, Chooichiro Asano** and Xianping LI*
}

\begin{abstract}
Qui [1] discussed the estimation problem of jump regression functions which were divided into eight types. $\mathrm{L}^{2}$-consistent estimates of two types of them were obtained. This paper studies further this topics and obtains $L^{2}-$ consistent estimates of the other four types. For the last two types, the authors also put forwards an estimate. A little numerical results are also given.
\end{abstract}

\section{Introduction}

Qiu [1] discussed the estimation problem of jump regression function $f(t)$.

$$
f(t)=g(t)+\sum_{i=1}^{p} d_{i} I_{\left(s_{i}, s_{i+1}\right)}(t), \quad t \in[0,1]
$$

where $g(t)$ is continuous, $p$ is the number of jumps, $\left\{s_{i}\right\}_{1}^{p}$ are jump points, $\left\{d_{i}-d_{i-1}\right\}_{1}^{p}$ are jump magnitudes, $d_{0}=0, s_{0}=0, s_{p+1}=1$. According to the information about the number of jumps, their locations and jump magnitudes, we can divide the jump regression functions into eight types. Qiu [1] obtained $L^{2}$-consistent estimates of the first two types, namely, $p,\left\{s_{i}\right\}_{1}^{p}$ are known, $\left\{d_{i}-d_{i-1}\right\}_{i=1}^{p}$ are known or unknown. It also discussed the case when the locations of jumps have some indeterminacy. In this paper, we will discuss the other types of jump regression functions. The idea of difference kernel estimate is suggested in Section 2 and with it we obtain a kind of $L^{2}$ and a.s. consistent estimates of jump locations and jump magnitudes of the (III) and (IV) jump regression functions. Using hypothesis testing, we gain a kind of $L^{2}$ and a.s. consistent estimates of the unknown parts of the (V) and (VI) jump regression functions in Section 3. We also put forwards a kind of estimates of the (VII) and (VIII) jump regression functions in this section. In the end, a little numerical results are given in Section 4.

\section{The (III) and (IV) Jump Regression Functions and Difference Kernel Estimate}

In this section, we will discuss the (III) and (IV) jump regression functions,

* Department of Statistics and Operations Research, Fudan University, Shanghai 200433, China.

** Research Institute of Fundamental Information Science, Kyushu University, Fukuoka 812, Japan. At present, Department of Information System Science, Soka University, Hachiouji, Tokyo, Japan. 
namely, the number of jumps is known, the jump locations are unknown and the jump magnitudes are known or unknown. If we can resolve the estimation problem of the (IV), we can resolve the (III) naturally. First of all, assume that regression function $f(t)$ has one jump point $0<\zeta<1$. Now we want to estimate $\zeta$ and the corresponding jump magnitude $C$ with observations $\left\{y_{i}\right\}_{1}^{n}$.

Suppose two kernels $K_{1}(x)$ and $K_{2}(x)$ satisfy:

(i) $K_{1}(x)=0$ for $x \bar{\epsilon}[0,1] ; \quad K_{2}(x)=0$ for $x \bar{\epsilon}[-1,0]$,

(ii) $K_{i}(x) \geq 0, \quad i=1,2, \quad x \in R^{1}$,

(iii) $\int_{1}^{-1} K_{i}(x) d x=1, \quad i=1,2$.

Define

$$
\begin{aligned}
& M_{n}(t)=\sum_{i=1}^{n} y_{i} \int_{t_{i-1}}^{t_{i}} h_{n}^{-1}\left[K_{1}\left(\frac{z-t}{h_{n}}\right)-K_{2}\left(\frac{z-t}{h_{n}}\right)\right] d z, \\
& 0<h_{n} \leq t \leq 1-h_{n}, \lim _{n \rightarrow \infty} n h_{n}=\infty, \lim _{n \rightarrow \infty} h_{n}=0, \\
& \left|M_{n}\left(t_{n}^{*}\right)\right| \triangleq \max _{h_{n} \leq t \leq 1-h_{n}}\left|M_{n}(t)\right| .
\end{aligned}
$$

In the condition of the continuities of $K_{i}(x), i=1,2, t_{n}^{*}$ exists. Of course, the points which have the property of $t_{n}^{*}$ would be more than one. In such a case, $t_{n}^{*}$ is the arbitrary one of them.

The $t_{n}^{*}$ and $M_{n}\left(t_{n}^{*}\right)$ are taken to be the estimates of $\zeta$ and $C$, respectively. We call them Difference Kernel Estimates. In the following, we will illustrate that $t_{n}^{*}$ and $M_{n}\left(t_{n}^{*}\right)$ are a.s. consistent estimates of $\xi$ and $C$.

Lemma 1. (1) Assume K(.) is boundary, $E\left|\varepsilon_{l}\right|^{p}<\infty, p \geq 2$.

$$
\delta_{\eta}=\max _{1 \leq i \leq n}\left(t_{i}-t_{i-1}\right)=O\left(n^{-1}\right), h_{n}=n^{-d}, 0<d<1-\frac{1}{p} .
$$

If regression function $g(t)$ is continuous on $[0,1]$, then for ${ }^{\forall} \in(0,1)$,

$$
g n(t) \triangleq \sum_{i=1}^{n} y_{i} \int_{t_{i-1}}^{t_{i}} h_{n}^{-1}\left[K\left(\frac{z-t}{h_{n}}\right)\right] d z \text { converges to } g(t) \text { almost surely. }
$$

(2) Furthermore, if $g(t) \in \operatorname{Lip}(1), K(.) \in \operatorname{Lip}(1)$, for $0<a \leq b \leq 1$,

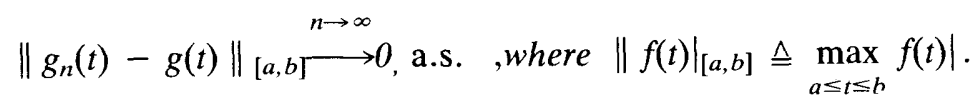

The proof can be found in Cheng and Ling [3]. From Lemma 1 (1), we have

$$
\lim _{n \rightarrow \infty} M_{n}(\xi)=C, \text { a.s. }
$$

\section{Denote}

$$
M_{n}(t)=M_{n}^{(1)}(t)-M_{n}^{(2)}(t),
$$


where

$$
\begin{aligned}
& M_{n}^{(j)}(t)=\sum_{i=1}^{n} y_{i} \int_{t_{i-1}}^{t_{i}} h_{n}^{-1} K_{j}\left(\frac{z-t}{h_{n}}\right) d z, \quad j=1,2 . \\
& \left\{\begin{array}{l}
f_{l}(t)=f(t), \quad t \in[0, \xi], \\
f_{2}(t)=f(t), \quad t \in[\xi, 1] .
\end{array}\right.
\end{aligned}
$$

We extend $f_{1}(t)$ to be $f_{1}^{*}(t)$, where the domain is $\left[-\frac{1-\xi}{2}, \frac{1+\xi}{2}\right]$, namely $f_{1}^{*}(t)=f_{1}(t)$ for $t \in[0, \xi]$. If $f_{1}^{*}(t) \in \operatorname{Lip}(1)$, then from Lemma $1(2)$,

$$
\left\|M_{n}^{*}(t)\right\|_{[0, \xi]} \leq\left\|M_{n}^{*(1)}(t)-f_{I}^{*}(t)\right\|_{[0, \xi]}+\left\|M_{n}^{*(2)}(t)-f_{I}^{*}(t)\right\|_{[0, \xi]} \stackrel{n \rightarrow \infty}{\longrightarrow} 0 \text {, a.s. }
$$

where $M_{n}^{*}(t)$ is defined like (2) with the regression $f *(t), M_{n}^{*}(t)=M_{n}^{*(1)}-M_{n}^{*(2)}(t)$, the meaning of $M_{n}^{*(i)}(t)$ is like $M_{n}^{(i)}(t), i=1,2$.

$$
\therefore\left\|M_{n}(t)\right\|_{\left(h_{n}, \xi-h_{n}\right)}=\left\|M_{n}^{*}(t)\right\|_{\left(h_{n}, \xi-h_{n}\right)} \leq\left\|M_{n}^{*}(t)\right\|_{[0, \xi]} \stackrel{n \rightarrow \infty}{\longrightarrow} 0, \text { a.s. }
$$

For the same reason,

$$
\left\|M_{n}(t)\right\|_{\left(\xi+h_{n}, 1-h_{n}\right)} \stackrel{n \rightarrow \infty}{\longrightarrow} 0, \text { a.s. }
$$

Combining the two parts above with (3), we have $\xi-h_{n} \leq t_{n}^{*} \leq \xi+h_{n}$, a.s., when $n$ is large enough. Then $\lim _{n \rightarrow \infty} t_{n}^{*}=\xi$, a.s.

Without loss of generality, we assume $C>0$.

Obviously,

$$
M_{n}\left(t_{n}^{*}\right)=\sum_{i=1}^{n} y_{i} \int_{t_{i-1}}^{t_{i}} h_{n}^{-1}\left[K_{I}\left(\frac{z-t_{n}^{*}}{h_{n}}\right)-K_{2}\left(\frac{z-t_{n}^{*}}{h_{n}}\right)\right] d z .
$$

$$
\begin{aligned}
& \sum_{i=1}^{n} \widetilde{y}_{i} h_{n}^{-1} \int_{t_{i-1}}^{t_{i}}\left[K_{I}\left(\frac{z-t_{n}^{*}}{h_{n}}\right)-K_{2}\left(\frac{z-t_{n}^{*}}{h_{n}}\right)\right] d z, \quad \xi-h_{n} \leq t_{n}^{*} \leq \xi, \\
& M_{n}\left(t_{n}^{*}\right) \geq \sum_{i=1}^{n} \underline{y}_{i} h_{n}^{-1} \int_{t_{i-1}}^{t_{i}}\left[K_{1}\left(\frac{z-t_{n}^{*}}{h_{n}}\right)-K_{2}\left(\frac{z-t_{n}^{*}}{h_{n}}\right)\right] d z, \quad \xi<t_{n}^{*} \leq \xi+h_{n}, \\
& \text { where } \quad \tilde{y}_{i}=y_{i}-C 1_{t_{i}>\xi}, \quad y_{i}=y_{i}+C 1_{t_{i} \leq \xi}, \quad i=\overline{1, n} \text {. } \\
& \text { If } f(t)-C 1_{t \geq \xi} \triangleq \widetilde{f}(t) \in \operatorname{Lip}(l) \text {, then } \\
& \begin{aligned}
\sum_{i=1}^{n} \widetilde{y}_{i} \int_{t_{i-1}}^{t_{i}} h_{n}^{-1}\left[K_{1}\left(\frac{z-t_{n}^{*}}{h_{n}}\right)\right. & \left.-K_{2}\left(\frac{z-t_{n}^{*}}{h_{n}}\right)\right] d z|\leq \max | \sum_{i=1}^{n} y_{i} \int_{t_{i-1}}^{t_{i}} h_{n}^{-1}\left[K_{1}\left(\frac{z-t}{h_{n}}\right)\right. \\
& \left.-K_{2}\left(\frac{z-t}{h_{n}}\right)\right] d z \mid \stackrel{n \rightarrow \infty}{\longrightarrow} 0, \text { a.s. }, \text { for } \frac{\xi}{2} \leq t \leq \frac{1+\xi}{2} .
\end{aligned}
\end{aligned}
$$


For the same reason,

$$
\begin{aligned}
& \left|\sum_{i=1}^{n} y_{i} t_{t_{i-1}}^{t_{i}} h_{n}^{-1}\left[K_{1}\left(\frac{z-t_{n}^{*}}{h_{n}}\right)-K_{2}\left(\frac{z-t_{n}^{*}}{h_{n}}\right)\right] d z\right| \stackrel{n \rightarrow \infty}{\longrightarrow} 0, \quad \text { a.s. } \\
& \therefore \lim _{n \rightarrow \infty} M_{n}\left(t_{n}^{*}\right) \geq 0, \quad \text { a.s. }
\end{aligned}
$$

and also

$$
\therefore \lim _{n \rightarrow \infty}\left|M_{n}\left(t_{n}^{*}\right)\right| \geq \lim _{n \rightarrow \infty}\left|M_{n}(\xi)\right|=C
$$

From (4) and (5), we have

$$
\lim _{n \rightarrow \infty} M_{n}\left(t_{n}^{*}\right) \geq C, \quad \text { a.s. }
$$

on the other hand, $M_{n}\left(\mathrm{t}_{n}^{*}\right)$ is not greater than

$$
\begin{gathered}
\sum_{i=1}^{n} \widetilde{y}_{i} h_{n}^{-1} \int_{t_{i-1}}^{t_{i}}\left[K_{1}\left(\frac{z-t_{n}^{*}}{h_{n}}\right)-K_{2}\left(\frac{z-t_{n}^{*}}{h_{n}}\right)\right] d z+C \sum_{i=1}^{n} \int_{t_{i-1}}^{t_{i}} h_{n}^{-1} K_{1}\left(\frac{z-t_{n}^{*}}{h_{n}}\right) \mathrm{dz}, \\
\text { for, } \xi-h_{n} \leq t_{n}^{*} \leq \xi ; \\
\sum_{i=1}^{n} \underline{y}_{i} h_{n}^{-1} \int_{t_{i-1}}^{t_{i}}\left[K_{1}\left(\frac{z-t_{n}^{*}}{h_{n}}\right)-K_{2}\left(\frac{z-t_{n}^{*}}{h_{n}}\right)\right] d z+C \sum_{i=1}^{n} \int_{t_{i-1}}^{t_{i}} h_{n}^{-1} K_{2}\left(\frac{z-t_{n}^{*}}{h_{n}}\right) \mathrm{dz}, \\
\text { for, } \quad \xi<t_{n}^{*} \leq \xi+h_{n}
\end{gathered}
$$

Obviously,

$$
\begin{aligned}
& \lim _{n \rightarrow \infty} \sum_{i=1}^{n} \int_{t_{i-1}}^{t_{i}} h_{n}^{-1} K_{j}\left(\frac{z-t_{n}^{*}}{h_{n}}\right) d z=1, \text { a.s. } j=1,2 . \\
& \therefore \lim _{n \rightarrow \infty} M_{n}\left(t_{n}^{*}\right) \geq C, \quad \text { a.s. }
\end{aligned}
$$

From (6) and (7), we have $\lim M_{n}\left(t_{n}^{*}\right)=C$, a.s. For the $C<0$ case, we can discuss similarly. Therefore we obtain:

THEOREM 1. $K_{i}(x)$ is boundary function and satisfy conditions (i) (iii), $i=1,2$, $E\left|\varepsilon_{1}\right|^{p}>\infty, p \geq 2, \delta_{n}=\max _{1 \leq i \leq n}\left(t_{i}-t_{i-1}\right)=O\left(n^{-1}\right), h_{n}=n^{-d}, 0<d<1-\frac{1}{p}$. $f_{i}(t) \in \operatorname{Lip}(1), K_{i}(x) \in \operatorname{Lip}(1), i=1,2$, then

(i) $t_{n}^{*}$ is a.s. consistent estimate of $\xi$;

(ii) Furthermore, if $f(t)-C l_{t>\xi} \in \operatorname{Lip}(1)$, then $M_{n}\left(t_{n}^{*}\right)$ is a.s. consistent estimate of $C$.

REMARK 1. Without loss of generality, assume $t_{i}-t_{i-1}=\frac{1}{n}, i=\overline{1, n}$ 
Then the number of items in (2) is $2 n h_{n}$.

If (i) $\left|K_{i}(x)\right| \leq M, i=2, \quad(M$ is an arbitrary positive constant. $)$,

(ii) $E\left|\varepsilon_{1}\right|^{2 q}<\infty, q>1$, then

$$
E\left|M_{n}\left(t_{n}^{*}\right)\right|^{2 q} \leqslant\left(2 n h_{n}\right)^{2 q-1} 2 n h_{n} E\left|y_{1}\right|^{2 q}\left(\frac{2 M}{n h_{n}}\right)^{2 q}=2^{2 q} E\left|y_{i}\right|^{2 q}(2 M)^{2 q}<\infty \text {. }
$$

Therefore $\left\{\left|M_{n}\left(t_{n}^{*}\right)\right|^{2}, n \geq 1\right\}$ are uniformly integrable. Then $M_{n}\left(t_{n}^{*}\right) \underset{n \rightarrow \infty}{\stackrel{L_{2}}{\longrightarrow}} C$, and $t_{n}^{*} \underset{n \rightarrow \infty}{\stackrel{L_{2}}{\longrightarrow}}$ $\xi$.

Thus, the spline correction theorem is true in this case, which was given in Qiu [1].

REMARK 2. The non-zero value domains of definition of $K_{I}(x)$ and $K_{2}(x)$ can be both extended to $[-T, T]$, where $T$ is an arbitrary positive constant. Namely, $K_{l}(x)=0$ when $x \bar{\in}[0, T] ; K_{2}(x)=0$ when $x \bar{E}[-T, 0]$.

Remark 3. The non-zero value domains of definition of $K_{l}(x)$ and $K_{2}(x)$ must be unilateral in order to guarantee $\lim M_{n}(\xi)=C$, a.s.

In fact

$$
\begin{aligned}
& M_{n}(\xi)= \sum_{i=1}^{n} y_{i} \int_{t_{i-1}}^{t_{i}} h_{n}^{-1}\left[K_{1}\left(\frac{z-\xi}{h_{n}}\right)-K_{2}\left(\frac{z-\xi}{h_{n}}\right)\right] d z \\
&= \sum_{i=1}^{n} \widetilde{y}_{i} \int_{t_{i-1}}^{t_{i}} h_{n}^{-1}\left[K_{1}\left(\frac{z-\xi}{h_{n}}\right)-K_{2}\left(\frac{z-\xi}{h_{n}}\right)\right] d z+C \int_{t_{r}}^{1} h_{n}^{-1}\left[K_{1}\left(\frac{z-\xi}{h_{n}}\right)\right. \\
&\left.-K_{2}\left(\frac{z-\xi}{h_{n}}\right)\right] d z \sim C \int_{t_{r}}^{1} h_{n}^{-1}\left[K_{1}\left(\frac{z-\xi}{h_{n}}\right)-K_{2}\left(\frac{z-\xi}{h_{n}}\right)\right] d z \\
&= C \int_{0}^{1} h_{n}^{-1} K_{1}\left(\frac{z-\xi}{h_{n}}\right) d z-C \int_{0}^{t_{r}} h_{n}^{-1} K_{1}\left(\frac{z-\xi}{h_{n}}\right) d z-C \int_{t_{r}}^{1} h_{n}^{-1} K_{2}\left(\frac{z-\xi}{h_{n}}\right) d z \\
&= C \int_{-\xi / h_{n}}^{(1-\xi) / h_{n}} K_{1}(y) d y-C \int_{-\xi / h_{n}}^{(1-\xi) / h_{n}} K_{1}(y) d y-C \int_{\left(t_{r}-\xi\right) / h_{n}}^{\left(1-\xi / h_{n}\right.} K_{2}(y) d y \\
& \stackrel{n \rightarrow \infty}{\longrightarrow} C-C \int_{-\infty}^{0} K_{1}(y) d y-C \int_{0}^{\infty} K_{(y) d y}
\end{aligned}
$$

where $t_{r}$ satisfy $t_{r}<\xi, t_{r+1} \geq \xi$. In order to guarantee $\lim M_{n}(\xi)=C$, a.s., it is necessary that $\int_{-\infty}^{0} K_{1}(y) d y=\int_{0}^{\infty} K_{2}(y) d y=0$. Because of the continuities of $K_{1}(y)$ and $K_{2}(y)$, we have $K_{1}(y) \equiv 0$ when $y \in(0, \infty)$. Namely, the non-zero value domains of definition of $K_{1}(x)$ and $K_{2}(x)$ are unilateral.

REMARK 4.

$$
\begin{gathered}
\sum_{i=1}^{n} \widetilde{y}_{i} h_{n}^{-1} \int_{t_{i-1}}^{t_{i}}\left[K_{1}\left(\frac{z-t_{n}^{*}}{h_{n}}\right)-K_{2}\left(\frac{z-t_{n}^{*}}{h_{n}}\right)\right] d z+\frac{C}{h_{n}} \int_{t_{r}}^{t_{n}^{*}+h_{n}} K_{1}\left(\frac{z-t_{n}^{*}}{h_{n}}\right) d z, \\
\xi-h_{n} \leq h_{\mathrm{n}}^{-1} \leq \xi
\end{gathered}
$$




$$
\begin{gathered}
M_{n}\left(t_{n}^{*}\right) \approx \sum_{i=1}^{n} \underline{y}_{i} h_{n}^{-1} \int_{t_{i-1}}^{t_{i}}\left[K_{1}\left(\frac{z-t_{n}^{*}}{h_{n}}\right)-K_{2}\left(\frac{z-t_{n}^{*}}{h_{n}}\right)\right] d z+\frac{C}{h_{n}} \int_{t_{n}^{*}-h_{n}}^{t_{r+1}} K_{2}\left(\frac{z-t_{n}^{*}}{h_{n}}\right) d z, \\
\xi<t_{n}^{*} \leq \xi+h_{n},
\end{gathered}
$$

where $t_{r}$ satisfy $t_{r}<\xi, t_{r+1} \geq \xi$, because of

$$
\begin{aligned}
& \frac{C}{H_{n}} \int_{t_{r}}^{t_{n}^{*}+h_{n}} K_{1}\left(\frac{z-t_{n}^{*}}{h_{n}}\right) d z \sim \frac{C}{h_{n}} \int_{\xi}^{t_{n}^{*}+h_{n}} K_{1}\left(\frac{z-t_{n}^{*}}{h_{n}}\right) d z=C \int_{\left(\xi-t_{n}^{*}\right) / h_{n}}^{1} K_{1}(y) d y, \\
& \frac{C}{h_{n}} \int_{t_{n}^{*}-h_{n}}^{t_{r+1}} K_{2}\left(\frac{z-t_{n}^{*}}{h_{n}}\right) d z \sim \frac{C}{h_{n}} \int_{t_{n}^{*}-h_{n}}^{\xi} K_{2}\left(\frac{z-t_{n}^{*}}{h_{n}}\right) d z=C \int_{-1}^{\left(\xi-t_{n}^{*}\right) / h_{n}} K_{y}(y) d y .
\end{aligned}
$$

Therefore if $\frac{\xi-t_{n}^{*}}{h_{n}} \stackrel{n \rightarrow \infty}{\longrightarrow} 0$, a.s., namely $\xi-t_{n}^{*}=o\left(h_{n}\right)$, then $M_{n}\left(t_{n}^{*}\right) \underset{n \rightarrow \infty}{\longrightarrow} C$.

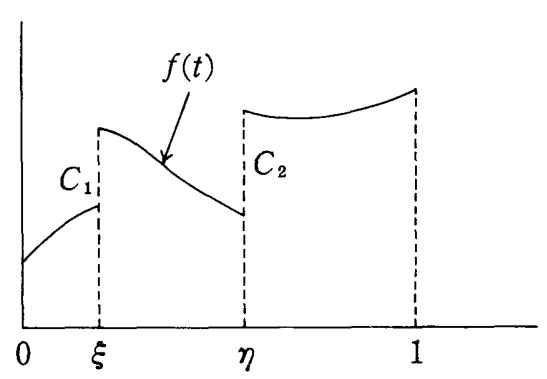

REMARK 5. Now, we discuss the case when the regression function has more then one jumps. Without loss of generality, assume the regression function has two jumps. Let $\left|M_{n}\left(t_{n}^{1}\right)\right|=\max _{h_{n} \leq t \leq 1-h_{n}}\left|M_{n}(t)\right|,\left|M_{n}\left(t_{n}^{2}\right)\right|=\max _{h_{n} \leq t \leq 1-h_{n}}\left|M_{n}(t)\right|$, where $\bar{t} \bar{\epsilon}\left(t_{n}^{1}-2 h_{n}\right.$, $\left.t_{n}^{l}+2 h_{n}\right)$ and $t_{n}^{1}$ and $t_{n}^{2}$ are arbitrary points which satisfy the relations above.

$t_{n}^{(1)}<t_{n}^{(2)}$ are order statistics of $t_{n}^{l}$ and $t_{n}^{2}$. We take $t_{n}^{(I)}, t_{n}^{(2)}$ as the estimates of $\zeta$ and $\eta$, the two jump points, respectively. Like the case of unique jump, $t_{n}^{(l)}, t_{n}^{(2)}, M_{n}\left(t_{n}^{(l)}\right)$, $M_{n}\left(t_{n}^{(2)}\right)$ are a.s. and $\mathrm{L}^{2}$ consistent estimates of $\xi$ and $\eta, C_{1}$ and $C_{2}$ respectively under some conditions.

Cheng [2] has the conclusion that

Lemma 2. Suppose $U_{l}, U_{2}, \ldots, U_{n}$ are i.i.d. random variables. $U_{1} \sim N\left(a, \sigma^{2}\right)$, $\left\{L \triangleq L_{n}\right\}_{I}^{\infty}$ are positive integers which satisfy the conditions that $\lim _{n \rightarrow \infty} \frac{L}{n}=0$ and $\lim _{n \rightarrow \infty} \frac{(\log n)^{2}}{L}=0$. Define 


$$
\begin{aligned}
\mathrm{V}_{\mathrm{m}} & =\left(\sum_{m-L+1}^{m} U_{i}-\sum_{m-2 L+1}^{m-L} U_{i}\right) / \sqrt{2 L}, \quad m=\overline{2 L, n} . \\
W_{n} & =\max \left\{\left[V_{m} \mid: m=\overline{2 L, n}\right\},\right. \\
A_{n}(x) & =\left\{2 \log \left(\frac{3 n}{2 L}-\right)\right\}^{-\frac{1}{2}}\left\{x+2 \log \left(\frac{3 n}{2 L}-3\right)+\frac{1}{2} \log \log \left(\frac{3 n}{2 L}-3\right)-\frac{1}{2} \log \pi\right\} .
\end{aligned}
$$

Then $\lim _{n \rightarrow \infty} P\left(\frac{W_{n}}{\sigma} \leq A_{n}(x)\right)=\exp \left(-2 e^{-x}\right), \quad-\infty<x<\infty$.

In our case, define

$$
\begin{aligned}
X_{m} & =\left(\sum_{m-L+1}^{m} Y_{i}-\sum_{m-2 L+1}^{m-1} i\right) / \sqrt{2 L}, \quad m=\overline{2 L, n} \\
& =\left(\sum_{m-L+1}^{m} \varepsilon_{i}-\sum_{m-2 L+1}^{m-L} \varepsilon_{i}\right) / \sqrt{2 L}+\left(\sum_{m-L+1}^{m} f\left(t_{i}\right)-\sum_{m-2 L+1}^{m-L} f\left(t_{i}\right)\right) / \sqrt{2 L} \\
& \triangleq X^{\prime}{ }_{m}+C_{m} .
\end{aligned}
$$

If $f(t)$ is differentiable on $[0,1]$ and $\left|f^{\prime}(t)\right| \leq M<\infty t \in(0,1)$, then

$$
\begin{aligned}
& \left|\sum_{m-L+1}^{m} f\left(t_{i}\right)-\sum_{m-2 L+1}^{m-L} f\left(t_{i}\right)\right| / \sqrt{2 L}=\left|\sum_{i=0}^{L-1}\left[f\left(t_{m-i}\right)-f\left(t_{m-L-i}\right)\right]\right| / \sqrt{2 L} \\
& \leq M \frac{L^{2}}{n} / \sqrt{2 L}=\frac{\sqrt{2}}{2} M n^{-1} L^{\frac{3}{2}} \\
& \xi_{n} \triangleq \sup \left\{\left|X_{m}\right|, m=\overline{2 L, n}\right\} \leq \sup \left\{\left|X_{m}^{\prime}\right|, m=\overline{2 L, n}\right\}+\frac{\sqrt{2}}{2} M n^{-1} L^{\frac{3}{2}} \\
& \triangleq \xi_{n}^{\prime}+\frac{\sqrt{2}}{2} M n^{-1} L^{\frac{3}{2}} .
\end{aligned}
$$

If $\varepsilon_{1} \sim N\left(0, \sigma^{2}\right)$, then according to Lemma 2,

$$
\lim _{n \rightarrow \infty} P\left(\frac{\xi_{n}^{\prime}}{\sigma} \geq A_{n}(x)\right)=1-\exp \left(-2 e^{-x}\right), \quad-\infty<x<\infty .
$$

Obviously,

$$
P\left(\frac{\xi_{n}}{\sigma} \geq A_{n}(x)\right) \leq P\left(\frac{\xi_{n}^{\prime}+\frac{\sqrt{2}}{2} M n^{-1} L^{\frac{3}{2}}}{\sigma} \geq A_{n}(x)\right)
$$




$$
\begin{gathered}
=P\left\{\frac{\left(\xi_{n}^{\prime}+\frac{\sqrt{2}}{2} M n^{-1} L^{\frac{1}{2}}\right) \sqrt{2 \log \left(\frac{3 n}{2 L}-3\right)}}{\sigma}\right. \\
\left.\geq x+2 \log \left(\frac{3 n}{2 L}\right)-3+\frac{1}{2} \log \log \left(\frac{3 n}{2 L}-3\right)-\frac{1}{2} \log \pi\right\} .
\end{gathered} .
$$

If $\quad \lim _{n \rightarrow \infty} \frac{\sqrt{2}}{2} M n^{-1} L^{\frac{3}{2}} \sqrt{2 \log \left(\frac{3 n}{2 L}-3\right)}=0$,

then $\lim _{n \rightarrow \infty} P\left(\frac{\xi_{n}^{\prime}+\frac{\sqrt{2}}{2} M n^{-1} L^{\frac{3}{2}}}{\sigma} \geq A_{n}(x)\right)=1-\exp \left(-2 e^{-x}\right), \quad x \in R^{l}$.

On the other hand, $\left|X_{m}\right| \geq\left|X_{m}^{\prime}\right|-\left|C_{m}\right| \geq\left|X_{m}^{\prime}\right|-\frac{\sqrt{2}}{2} M n^{-1} L^{\frac{3}{2}}$.

$$
\begin{aligned}
& \therefore \xi_{n} \geq \xi_{n}^{\prime}-\frac{\sqrt{2}}{2} M n^{-1} L^{\frac{3}{2}} . \\
& \therefore P\left(\frac{\xi_{n}}{\sigma} \geq A_{n}(x)\right) \geq P\left(\frac{\xi_{n}^{\prime}-\frac{\sqrt{2}}{2} M n^{-1} L^{\frac{3}{2}}}{\sigma} \leq A_{n}(x)\right) .
\end{aligned}
$$

Under the condition of (9), we have

$$
\lim _{n \rightarrow \infty} P\left(\frac{\xi_{n}^{\prime}-\frac{\sqrt{2}}{2} M n^{-1} L^{\frac{3}{2}}}{\sigma} \geq A_{n}(x)\right)=1-\exp \left(-2 e^{-x}\right), \quad x \in R^{I} .
$$

Combining (8), (10), (11) and (12), we have

$$
\lim _{n \rightarrow \infty} P\left(\frac{\xi_{n}}{\sigma} \geq A_{n}(x)\right)=1-\exp \left(-2 e^{-x}\right), \quad x \in R^{I} .
$$

From this, we obtain:

THEOREM 2. Suppose $\varepsilon_{I} \sim N\left(0, \sigma^{2}\right),\left\{L=L_{n}\right\}_{1}^{\infty}$ are positive integers which satisfy (i) $\lim _{n \rightarrow \infty} L / n=0$, (ii) $\lim _{n \rightarrow \infty}(\log n)^{2} / L=0$.

(iii) $\lim _{n \rightarrow \infty} n^{-1} L^{\frac{3}{2}} \sqrt{\log \left(\frac{3 n}{2 L}-3\right)}=0 . f(t)$ is differentiable over $[0,1]$ and $\left|f^{\prime}(t)\right| \leq M<$ $\infty, t \stackrel{n \rightarrow \infty}{\in}(0,1)$. Then

$$
\lim _{n \rightarrow \infty} P\left(\frac{\xi_{n}}{\sigma} \leq A_{n}(x)\right)=\exp \left(-2 e^{-x}\right), \quad x \in R^{1}
$$


Therefore, we can test the hypothesis $H_{0}: \quad C=0 \leftrightarrow H_{l}: C \neq 0$ under the condition that $f(t)-C l_{t} \xi$ is differentiable over $[0,1]$ and its derivative is boundary. The concrete test procedure is that for significance level $\alpha \in(0,1), x(\alpha)=-$ $\log \left(-\frac{1}{2} \log (1-\alpha)\right)$ is the solution of the equation $\exp \left(-2 e^{-x}\right)=1-\alpha$ and we reject $H O$ if and only if $\xi_{n}>\sigma A_{n}(x(\alpha))$. This test has the asymptotic significance level $\alpha$.

The discussion about $\beta(\theta)$, interval estimate of jump point, Th2, Th4, Th5 and many others in Cheng [2] are right in our case after some simple remedies.

\section{Estimation of Other Four Types of Jump Regression Functions}

First of all, we discuss the (v) and (VI) jump regression functions. In this case, the number of jumps is unknown, but jumps can only take place at m positions $0<\xi_{1}<\xi_{2}$ $<\ldots<\xi_{\mathrm{m}}<1$ on $[0,1]$.

Suppose there are $\eta_{\mathrm{i}}$ observations on Gauss' $\left[0, \xi_{\mathrm{i}}\right], i=\overline{1, m}$ Let's investigate the r.v. array

$$
\left\{\left(\varepsilon_{n 1+i}-\varepsilon_{n 1-i}\right) /\left[n^{\varepsilon}\right]^{\frac{1}{2}} \sqrt{2} \sigma, i=\overline{1,\left[n^{\varepsilon}\right]}\right\} .
$$

Firstly $\sum_{i=1}^{\left[n^{\varepsilon}\right]} \operatorname{var}\left[\frac{\varepsilon_{n l+i}-\varepsilon_{n l-i}}{\left[n^{\varepsilon}\right]^{\frac{l}{2}} \sqrt{2} \sigma}\right]=1$, and secondly, for ${ }^{\forall} \eta>0$,

$$
\begin{aligned}
& \lim _{n \rightarrow \infty} \sum_{\mathrm{i}=1}^{\left[n^{\varepsilon}\right]} \int x^{2} d F_{n i \geq \eta}(x), \quad\left(F_{n i}(x) \text { is d.f. of } \frac{\varepsilon_{n l+i}-\varepsilon_{n 1-i}}{\left[n^{\varepsilon}\right]^{\frac{1}{2}} \sqrt{2} \sigma}\right) \\
& =\lim _{n \rightarrow \infty|y| \geq\left[n^{\varepsilon}\right]^{\frac{1}{2}} \eta} f y^{2} d G(y),\left(G(y) \text { is d.f. of } \frac{\varepsilon_{2}-\varepsilon_{1}}{\sqrt{2} \sigma}\right)=0 .
\end{aligned}
$$

The condition for last equation is that $\varepsilon_{1}$ has the second order moment.

Therefore the r.v. array above satisfy the Linderberg conditions, and

$$
\eta_{n} \triangleq \sum_{i=1}^{\left[n_{\varepsilon}\right]} \frac{\varepsilon_{n 1+i}-\varepsilon_{n 1-i}}{\left[n^{\varepsilon}\right]^{\frac{1}{2}} \sqrt{2} \sigma} \stackrel{D}{\longrightarrow} N(0,1)
$$

Now, make the hypothesis at $\xi_{1}$ that $H_{0}$ : there is no jump at $\zeta_{1} \leftrightarrow H_{l}: C \neq 0$ is the jump magnitude at $\xi_{1}$. We take the following procedure to test this hypothesis. For level $\alpha_{\mathrm{n}}, C_{\alpha n / 2}$ is generated by $N(0,1)$, namely $P\left(|X|>C_{\alpha n / 2}\right)=\alpha_{n}$, where $X \sim N(0$, 1). We reject $H_{0}$, if

$$
\left|\xi_{n}\right| \triangleq \frac{1}{\left[n^{\varepsilon}\right]^{\frac{1}{2}} \sqrt{2} \sigma}\left|\sum_{i=1}^{\left[n^{\varepsilon}\right]}\left(Y_{n I+i}-Y_{n I-i}\right)\right| \geq \mathrm{C}_{\alpha n / 2} .
$$

If $H_{0}$ is true 


$$
\begin{gathered}
\frac{1}{\left[n^{\varepsilon}\right]^{\frac{1}{2}} \sqrt{2} \sigma}\left|\sum_{i=1}^{\left[n^{\varepsilon}\right]}\left(f\left(t_{n l+i}\right)-f\left(t_{n l-i}\right)\right)\right| \geq \frac{1}{\left[n^{\varepsilon}\right]^{\frac{1}{2}} \sqrt{2} \sigma} \sum_{i=1}^{\left[n^{\varepsilon}\right]}\left|f^{\prime}\left(p_{i}\right)\right|\left(t_{n 1+i}-t_{n 1-i}\right) \\
\leq \frac{M\left[n^{\varepsilon}\right]\left(\left[n^{\varepsilon}\right]+\right)}{\left[n^{\varepsilon}\right]^{\frac{1}{2}} \sqrt{2} \sigma}=O\left(n^{(3 \varepsilon-2) / 2}\right),
\end{gathered}
$$

where $t_{n 1-i} \leq p_{i} \leq t_{n 1+i}, i=\overline{1,\left[n^{\varepsilon}\right]},\left|f^{\prime}(t)\right| \leq M, \quad t \in(0,1)$.

If $\varepsilon<2 / 3$, then $\lim _{n \rightarrow \infty} \frac{1}{\left[n^{\varepsilon}\right]^{\frac{3}{2}} \sqrt{2}} \sum_{i=1}^{\left[n^{k}\right]}\left[f\left(t_{n 1+i}\right)-f\left(t_{n 1-i}\right)\right]=0$.

Therefore

$$
\frac{1}{[n \varepsilon]^{\frac{3}{2}} \sqrt{2}} \sum_{i=1}^{\left[n^{t}\right]}\left[Y_{n 11 i}-Y_{n 1-i}\right] \stackrel{D}{\longrightarrow} N(0,1) \text {. }
$$

The probability of the first error: $r_{n} \triangleq P_{H_{0}}\left(\left|\xi_{n}\right| \geq C_{\alpha n / 2}\right)$. If $\lim _{n \rightarrow \infty} \alpha_{n}=0$, then

$$
\lim _{n \rightarrow \infty} C_{\alpha n / 2}=\infty
$$

$\lim _{n \rightarrow \infty} P_{H_{0}}\left(\left|\xi_{n}\right| \geq C_{\alpha n / 2}\right) \leq \lim _{n \rightarrow \infty} P_{H_{0}}\left(\left|\xi_{n}\right| \geq N\right)=P(|X| \geq N)$, where $N$ is an arbitrarily set positive number.

$$
\therefore \lim _{n \rightarrow \infty} r_{n}=0
$$

The probability of the second error

$S_{n} \triangleq 1-P_{H_{l}}\left(\left|\xi_{n}\right| \geq C_{\alpha n / 2}\right)=1-\left\{P_{H_{1}}\left(\eta_{n} \geq C_{\alpha n / 2}-d_{n}\right)+P_{H_{j}}\left(\eta_{n} \leq-C_{\alpha n / 2}-d_{n}\right)\right\}$,

for $\quad d_{n}=\frac{1}{\left[n^{\varepsilon}\right]^{\frac{1}{2}} \sqrt{2} \sigma} \sum_{i=1}^{\left[n^{\varepsilon}\right]}\left[f\left(t_{n 1+i}\right)-f\left(t_{n 1-i}\right)\right]=\frac{\left[n^{\varepsilon}\right]}{\left[n^{\varepsilon}\right]^{\frac{1}{2}} \sqrt{2} \sigma} C+O\left(n^{(3 \varepsilon-2) / 2}\right)=O\left(n^{\varepsilon / 2}\right)$.

If $\lim _{n \rightarrow \infty} \frac{C_{\alpha n} / 2}{d_{n}}=0$, then $\lim _{n \rightarrow \infty} S_{n}=0$.

We can make tests at other points similarly and take the number $a_{n}$ of rejecting $H_{O}$ in all $m$ tests as the estimate of the number of jumps $p$ and the corresponding positions as the estimates of the jump positions. If $H_{1}$ is received at $\varepsilon_{\mathrm{j}}$, then we use

$$
\frac{1}{\left[n^{\varepsilon}\right]} \sum_{i=1}^{\left[n^{\varepsilon}\right]}\left[Y\left(t_{n j+i}\right)-Y\left(t_{n j-i}\right)\right]
$$

as the estimate of jump magnitude at $\xi_{j}$.

THEOREM 3. If (i) $\varepsilon^{1}$ has the second order moment $\sigma^{2}$ known,

(ii) $\left|f^{\prime}(t)\right| \leq M<\infty, t \in(0,1)$, (iii) $\varepsilon<2 / 3$, and 
(iv) $\lim _{n \rightarrow \infty} \alpha_{n}=0$ and $\lim _{n \rightarrow \infty} \frac{C_{\alpha n / 2}}{n^{\varepsilon / 2}}=0$, then $a_{n} \stackrel{L^{2}}{\longrightarrow} p$.

Furthermore, if

(v) $\varepsilon_{1} \sim N\left(0, \sigma^{2}\right), \quad(v i) \sum_{n=1}^{\infty} \exp \left\{-\frac{\left(C \alpha_{n / 2}-1\right)^{2}}{2}\right\}<\infty$, then $a_{n} \stackrel{\text { a.s. }}{\rightarrow} p$.

Proof. Denote $a_{n} \triangleq p_{n}+q_{n}$ where $P_{n}$ is the number of rejecting $H_{0}$ at $p$ points which really have jumps and $q_{n}$ is the number of rejecting $H_{O}$ at $m-p$ points which actually have no jumps.

$r_{n j}$ denote the probability of the (I) error at $\xi j$. From the analysis about two types of errors above, we have $\lim r_{n i}=0, i=\overline{1, m}$, under the conditions of (i)-(iv). Without loss of generality, assume the first $p$ points of $\left\{\xi_{j}\right\}_{1}^{m}$ really have jumps and the last $\mathrm{m}-\mathrm{p}$ points have no.

Then $P\left(q_{n}=0\right)=\prod_{i=p+1}^{m}\left(1-r_{n i}\right) \stackrel{n \rightarrow \infty}{\longrightarrow} 1$,

$$
E q_{n}^{2}=1^{2} P\left(q_{n}=1\right)+2^{2} P\left(q_{n}=2\right)+\cdots+(m-p)^{2} P\left(q_{n}=m-p\right) \stackrel{n \rightarrow \infty}{\longrightarrow} 0 .
$$

$\therefore q_{n} \stackrel{L^{2}}{\longrightarrow}$ 0. Similarly, $p_{n} \stackrel{L^{2}}{\longrightarrow} 0$.

$$
\therefore a_{n}=p_{n}+q_{n} \stackrel{L^{2}}{\longrightarrow} p \text {. }
$$

If $\varepsilon_{I} \sim N\left(0, \sigma^{2}\right)$, then $r_{n i}=P\left(\left|\eta_{n}+d_{n i}\right| \geq C_{\alpha n / 2}\right)$

$=P\left(\eta_{n} \geq C_{\alpha n / 2}-d_{n i}\right)+P\left(\eta_{n} \leq-C_{\alpha n 2} \leq d_{n i}\right)$

$\leq \frac{1}{\sqrt{2 \pi}} \frac{1}{C_{\alpha n / 2}+d_{n i}} \exp \left\{-\frac{\left.C_{\alpha n / 2}-d_{n i}\right)^{2}}{2}\right\}+\frac{1}{\sqrt{2} \pi} \frac{1}{C_{\alpha n / 2}+d_{n i}} \exp \left\{-\frac{\left(C_{\alpha n / 2}+d_{n i}\right)^{2}}{2}\right\}$

$\leq K \exp \left\{-\frac{\left(C_{a n / 2}-1\right)^{2}}{2}\right\}$

where $d_{n i}=\frac{1}{\left[n^{\varepsilon}\right]^{\frac{1}{2}} \sqrt{2} \sigma} \sum_{i=1}^{\left[n^{\varepsilon}\right]}\left[f\left(t_{n_{i}+j}\right)-f\left(t_{n_{i}-j}\right)\right]$, and $K$ is a positive constant.

For $0<\delta<1$,

$$
\begin{aligned}
\sum_{n=1}^{\infty} P\left(q_{n}>\delta\right) & =\sum_{n=1}^{\infty}\left(1-\prod_{i=p+1}^{m}\left(1-r_{n i}\right)\right) \\
& =\sum_{n=1}^{\infty} \sum_{i=p+1}^{m} r_{n i}-\sum_{n=1}^{\infty} \sum_{i, j=p+1}^{\infty} r_{n i} r_{n j}+\cdots+(-1)^{m-p+1} \sum_{n=1}^{\infty} \prod_{i=p+1}^{m} r_{n i}
\end{aligned}
$$


If we can illustrate that

$$
\sum_{n=1}^{\infty} \sum_{i=p+1}^{m} r_{n i}<\infty \text { then } \sum_{n=1}^{\infty} P\left(q_{n}>\delta\right)<\infty,
$$

but

$$
\sum_{n=1}^{\infty} \sum_{i=p+1}^{m} r_{n i} \leq(m-p) K \sum_{n=1}^{\infty} \exp \left\{-\frac{\left(C_{\alpha n / 2}-1\right)^{2}}{2}\right\}
$$

Therefore, according to Borel-Cantelli Lemma, $q_{n} \stackrel{\text { a.s. }}{\longrightarrow} 0$ under the condition of (iv). Similarly, $p_{n} \stackrel{\text { a.s. }}{\longrightarrow}$ p.

$$
\therefore a_{n}=p_{n}+q_{n} \stackrel{\text { a.s. }}{\longrightarrow} \mathrm{p} \text {. }
$$

The a.s. and $L^{2}$ consistent estimate $a_{n}$ of $p$ discussed above requires $\sigma^{2}$ is known. This condition is very severe. In the following, we will construct $L^{2}$-consistent estimate of $p$ under the condition that $\varepsilon_{1} \sim N\left(0, \sigma^{2}\right)$ and $\sigma^{2}$ is unknown.

Let

$$
\begin{aligned}
& X_{i}=\varepsilon_{n 1+i}-\varepsilon_{n 1-i,}, \quad X_{i}^{\prime}=Y_{n 1+i}-Y_{n 1-i}, \\
& \mathrm{~d}_{\mathrm{i}}=f\left(t_{n 1+i}\right)-f\left(t_{n 1-i}\right), \quad i=\overline{1,\left[n^{\natural}\right]}, \\
& \bar{X}=\frac{1}{\left[n^{i}\right]} \sum_{i=1}^{\left[n^{t}\right]} X_{i}, \quad \bar{X}^{\prime}=\frac{1}{\left[n^{\varepsilon}\right]} \sum_{i=1}^{\left[n^{t}\right]} X_{i}^{\prime}, \quad \bar{d}=\frac{1}{\left[n^{t}\right]} \sum_{i=1}^{\left[n^{\varepsilon}\right]} d_{i} . \\
& S_{\left[n^{i}\right]}^{2}=\frac{1}{\left[n^{k}\right]} \sum_{i=1}^{\left[n^{t}\right]}\left(X_{i}-\bar{X}\right)^{2} S_{\left[n^{\varepsilon}\right]}^{2}=\frac{1}{\left[n^{i}\right]} \sum_{i=1}^{\left[n^{t}\right]}\left(X_{i}-\overline{X^{\prime}}\right)^{2} .
\end{aligned}
$$

Then $\bar{X} \sim N\left(0, \frac{2 \sigma^{2}}{\left[n^{t}\right]}\right)$, and it is independent of $S_{\left[n^{t}\right]}^{2}$. Making hypothesis at $\xi_{1}$ that $H_{0}$ : there is no jump at $\xi_{1} \leftrightarrow H_{l}$ : there is jump at $\xi_{1}$ and the jump magnitude is $C \neq 0$.

We can see

$$
\begin{aligned}
S_{\left[n^{\natural}\right]}^{2} & \left.=\frac{1}{\left[n^{\varepsilon}\right]} \sum_{i=1}^{\left[n^{\varepsilon}\right]} X_{i}^{\prime 2}-\overline{X^{\prime}}=\frac{1}{\left[n^{\varepsilon}\right]} \sum_{i=1}^{\left[n^{\varepsilon}\right]}\left(X_{i}+d_{i}\right)^{2}-\bar{X}+\bar{d}\right)^{2} \\
& =\frac{1}{\left[n^{\varepsilon}\right]} \sum_{i=1}^{\left[n^{\varepsilon}\right]} X_{i}^{2}+\frac{2}{\left[n^{\varepsilon}\right]} \sum_{i=1}^{\left[n^{\varepsilon}\right]} X_{i} d_{i}+\frac{1}{\left[n^{\varepsilon}\right]} \sum_{i=1}^{\left[n^{\varepsilon}\right]} d_{i}^{2}-(\bar{X}+\bar{d})^{2},
\end{aligned}
$$

When $H_{0}$ is true, it is easy to know that

$$
\lim _{n \rightarrow \infty}(\bar{X}+\bar{d})^{2}=0 \text {, a.s., } \lim _{n \rightarrow \infty} \frac{2}{\left[n^{\varepsilon}\right]} \sum_{i=1}^{\left[n^{\varepsilon}\right]} d_{i}^{2}=0 \text { and } \lim _{n \rightarrow \infty} \frac{2}{\left[n^{\varepsilon}\right]} \sum_{i=1}^{\left[n^{\varepsilon}\right]} X_{i} d_{i}=0 \text {, a.s. }
$$

Thus $\lim _{n \rightarrow \infty} S_{\left[n^{t}\right]}^{\prime 2}=2 \sigma^{2}$, a.s. 
When $H_{1}$ is true, let $D_{i}-c=d_{i}^{\prime}, i=\overline{1,\left[n^{\xi}\right]}, \bar{d}-c=\bar{d}^{\prime}$,

Then

$$
\lim _{n \rightarrow \infty} \sup _{1 \leq i \leq\left[n^{*}\right]}\left|d_{i}^{\prime}\right|=0 \text { and } \lim _{n \rightarrow \infty} \bar{d}^{\prime}=0 \text {. }
$$

Similarly

$$
\begin{aligned}
S_{\left[n^{\varepsilon}\right]}^{2} & =\frac{1}{\left[n^{\varepsilon}\right]} \sum_{i=1}^{\left[n^{\varepsilon}\right]}\left(X_{i}^{\prime}-\overline{X^{\prime}}\right)^{2}=\frac{1}{\left[n^{\varepsilon}\right]} \sum_{i=1}^{\left[n^{\varepsilon}\right]}\left(X_{i}+d_{i}-\bar{X}-\bar{d}\right)^{2} \\
& =\frac{1}{\left[n^{\varepsilon}\right]} \sum_{i=1}^{\left[n^{\varepsilon}\right]}\left(X_{i}+d_{i}^{\prime}-\bar{X}-\overline{d^{\prime}}\right)^{2} \\
& =\frac{1}{\left[n^{\varepsilon}\right]} \sum_{i=1}^{\left[n^{\varepsilon}\right]} X_{i}^{2}+\frac{2}{\left[n^{\varepsilon}\right]} \sum_{i=1}^{\left[n^{\varepsilon}\right]} X_{i} d_{i}^{\prime}+\frac{1}{\left[n^{\varepsilon}\right]} \sum_{i=1}^{\left[n^{\varepsilon}\right]} d_{i}^{\prime 2}-\left(\bar{X}+\overline{d^{\prime}}\right)^{2} .
\end{aligned}
$$

As the case when $H_{0}$ is true, we can obtain $\lim _{n \rightarrow \infty} S_{\left[n^{k}\right]}^{2}=2 \sigma^{2}$, a.s.

Let $\zeta_{n}=\frac{1}{\left[n^{\varepsilon}\right]^{\frac{1}{2}}} \sum_{i=1}^{\left[n^{\varepsilon}\right]}\left(Y_{n 1+i}-Y_{n 1-i}\right)$. As for the above hypothesis test problem, we can take the following procedure: rejecting $H_{o}$ when $\left|\xi_{n}\right| \geq \beta_{n}$, where $\left\{\beta_{n}\right\}$ is a series of positive real numbers which satisfy $\lim _{n \rightarrow \infty} \beta_{n}=\infty$.

When $H_{O}$ is true, we can see that

$$
\frac{1}{\left.\left[n^{\varepsilon}\right]^{\frac{1}{2}} S_{\left[n^{\varepsilon}\right.}^{\prime}\right]}\left|\sum_{i=1}^{\left[n^{\varepsilon}\right]}\left[f\left(t_{n 1+i}\right)-f\left(t_{n 1-i}\right)\right]\right|=0\left(n^{\frac{3 \varepsilon-2}{2}}\right) \text {, a.s. }
$$

If $\varepsilon<2 / 3$, then

$$
\left.\lim _{n \rightarrow \infty} \frac{1}{\left[n^{\varepsilon}\right]^{\frac{1}{2}} S_{\left[n^{\epsilon}\right]}^{\prime}} \sum_{i=1}^{\left[n^{\varepsilon}\right]} f\left(t_{n 1+i}\right)-f\left(t_{n 1-i}\right)\right]=0, \text { a.s. }
$$

The (first) error probability $r_{n} \triangleq P_{H_{0}}\left(\left|\xi_{n}\right| \geq \beta_{n}\right)$

$$
\lim _{n \rightarrow \infty} r_{n} \leq \lim _{n \rightarrow \infty} P_{H_{0}}\left(\left|\xi_{n}\right|>N\right)=2 \phi(-N),
$$

where $N$ is an arbitrary positive number. Therefore $\lim _{n \rightarrow \infty} r_{n}=0$.

The second error probability

$$
\begin{aligned}
\omega_{n} & \triangleq P_{H_{l}}\left(\left|\xi_{n}\right| \geq \beta_{n}\right)=E\left\{P_{H_{l}}\left(\left|\xi_{n}\right|<\left.\beta\right|_{n} S_{\left[n^{\varepsilon}\right]}^{\prime}\right)\right\} \\
& =E\left\{P_{H_{l}}\left(\frac{S_{\left[n^{\ddagger}\right]}^{\prime}}{\sqrt{2} \sigma}\left(-\beta_{n}-h_{n}\right) \leq \frac{\left[n^{\varepsilon}\right]^{\frac{1}{2}} X}{\sqrt{2} \sigma} \leq\left(\beta_{n}-h_{n}\right) \frac{S_{\left[n^{\varepsilon}\right]}^{\prime}}{\sqrt{2} \sigma} \mid S_{\left[n^{\varepsilon}\right]}^{\prime}\right]\right\},
\end{aligned}
$$

where

$$
\left.h_{n}=\frac{1}{\left[n^{\varepsilon}\right]^{\frac{1}{2}} S_{\left[n^{\varepsilon}\right]}^{\prime}} \sum_{i=1}^{\left[n^{\varepsilon}\right]} f\left(t_{n 1+i}\right)-f\left(t_{n 1-i}\right)\right]=\frac{1}{\left[n^{\varepsilon}\right]^{\frac{1}{2}} S_{\left[n^{\varepsilon}\right]}^{\prime}} \mathrm{C}+\mathrm{O}\left(n^{\frac{3 \varepsilon-2}{2}}\right)=O\left(n^{\varepsilon / 2}\right) \text {, a.s. }
$$


If $\lim _{n \rightarrow \infty} n^{-\varepsilon / 2} \beta_{n}=0$, then

$$
P_{H_{t}}\left[\frac{S_{\left[n^{\varepsilon}\right]}^{\prime}}{\sqrt{2} \sigma}\left(-\beta_{n}-h_{n}\right) \leq \frac{\left[n^{\varepsilon}\right]^{\frac{1}{X}} \bar{X}}{\sqrt{2} \sigma} \leq\left(\beta_{n}-h_{n}\right) \frac{S_{\left[n^{\varepsilon}\right]}^{\prime}}{\sqrt{2} \sigma} \mid S_{\left[n^{\natural}\right]}^{\prime}\right], \stackrel{n \rightarrow \infty}{\longrightarrow} 0 \text {, a.s. }
$$

According to Lebesque Control and Convergence Theorem, $\lim _{n \rightarrow \infty} W_{n}=0$.

We can make hypothesis tests similarly at $\xi_{2}, \ldots, \xi_{\mathrm{m}}$ and use the number of rejecting $H_{o}$ of all $m$ tests as the estimate of $p$. It is easy to prove like theorem 3 that it is a $L^{2}$-consistent estimate of $p$.

In the following, we put forwards a type of estimates of the (VII) and (VIII) jump regression functions, where the number of jumps and jump locations are unknown.

Let

$$
X_{i}=\left|\frac{1}{\left[n^{\varepsilon}\right]} \sum_{j=1}^{\left[n^{\varepsilon}\right]}\left(y_{\left[n^{\varepsilon}\right]+i+j}^{-y}{ }_{\left[n^{\varepsilon}\right]+i-j}\right)\right|, \quad i=\overline{1,\left(n-2\left[n^{\varepsilon}\right]\right)},
$$

where $0<\varepsilon<1$ is a constant. $\left.X_{(1)} \geqslant X_{(2)} \geqslant \ldots \geqslant X_{\left(n-2\left[n^{\varepsilon}\right]\right.}\right)$ are order statistics of $\left\{X_{i}\right\}_{l}{ }^{\left(n-2\left[n^{k}\right]\right)}$. We devide them into two parts at the position where the difference between two neighbouring order statistics is maximal. Without loss of generality, the two parts are supposed as $X_{(1)} \geqslant X_{(2)} \geqslant \cdots \geqslant X_{(L)}$ and $X_{(L+1)} \geqslant X_{(L+2)} \geqslant \cdots \geqslant$ $X_{\left(n-\left[n^{t}\right]\right.}$, where $X_{(L)}-X_{(L+1)}=\max _{l \leq i \leq n-n\left[n^{\varepsilon}\right]-1}\left(X_{(i)}-X_{(i+1)}\right)$. The corresponding statistics of $\left\{X_{(i)}\right\}_{I}^{L}$ are $X_{i_{1}+1}, \cdots, X_{i_{1}+j_{1}} ; X_{i_{2}+1}, \cdots, X_{i_{2}+j_{2}} ; \cdots ; X_{i_{k}+1}, \cdots, X_{i_{k}+j_{k}}$ where $i_{s}+j_{s}<i_{s+1}, s=\overline{1, k-1}, \sum_{s=1}^{k}=L$. Select a representative in every group of statistics above which corresponding order statistics is ahead of other's in its group. We denote these representatives as $X_{p_{l}}, X_{p_{2}}, \cdots, X_{p_{k}}$, where $i_{L}+1 \leqslant p_{L} \leqslant i_{L}+j_{L}, L=$ $\overline{1, k}$. Then we take $\mathbf{k}$ as the estimate of the number of jumps, $\mathbf{t}_{\left[n^{\varepsilon}\right]}+{ }_{p_{I}}, t_{\left[n^{\varepsilon}\right]}+{ }_{p_{2}}, \cdots$, $\underset{t_{\left[n^{\varepsilon}\right]}+p_{k}}{1} p_{\left.k n^{\varepsilon}\right]}$ as the estimates of jump positions and $\frac{1}{\left[n^{\varepsilon}\right]} \sum_{j=1}^{\left[n^{\varepsilon}\right]}\left(y_{\left[n^{\varepsilon}\right]+p_{1}+j}-y_{\left[n^{\varepsilon}\right]+p_{l}-j}\right), \cdots$, $\frac{1}{\left[n^{\varepsilon}\right]} \sum_{j=1}^{\left[n^{\varepsilon}\right]}\left(y_{\left[n^{\varepsilon}\right]+p_{k}+j}-y_{[n} \varepsilon_{\left[+p_{k}-j\right.}\right)$ as the estimates of corresponding jump magnitudes.

\section{Some Numerical Results}

For the Difference Kernel Estimate proposed in section 2, we made some simulations after replacing $M_{n}(t)$ in (3) by

$$
\widetilde{M}_{n}(t)=\frac{1}{n h_{n}} \sum_{j=1}^{n} y_{i}\left[K_{l}\left(\frac{{ }^{t} i^{-t}}{h_{n}}\right)-K_{2}\left(\left(\frac{{ }^{t} i^{-t}}{h_{n}}\right)\right]\right. \text {. }
$$

The regression function used is $f(x)=x^{2}+3 x+201_{[0.5,1]} \varepsilon_{1} \sim N(0,1), h_{n}=1 / 1 n n$. Use scanning method to obtain $t_{n}^{*}$. The number of scannings is $B$.

Firstly, we choose

$$
K(x)=\left\{\begin{array}{l}
1, x \in[0,1] \\
0, \text { when } x \text { at other points; } \mathrm{n}=50 \\
-1, x \in[-1,0]
\end{array}\right.
$$


where $K(x)=K_{1}(x)-K_{2}(x)$. The simulation results with varied $B$ are:

\begin{tabular}{cccccc}
\hline$B$ & 10 & 20 & 50 & 100 & 200 \\
\hline$t_{s e}$ & 0.5 & 0.45 & 0.52 & 0.49 & 0.495 \\
\hline$\widetilde{M}_{s 0}\left(t_{s p}^{*}\right)$ & 18.153 & 18.285 & 20.069 & 21.448 & 21.448 \\
\hline
\end{tabular}

Then $\mathrm{I}$ set $B=10$. The results with the same kernel and varied $n$ are:

\begin{tabular}{cccccc}
\hline$n$ & 50 & 100 & 200 & 500 & 1000 \\
\hline$t_{n}^{*}$ & 0.5 & 0.5 & 0.5 & 0.5 & 0.5 \\
\hline$\widetilde{M}_{n}\left(t_{n}^{*}\right)$ & 18.153 & 19.065 & 20.211 & 20.087 & 20.260 \\
\hline
\end{tabular}

Afterwards, we choose

$$
K(x)=\left\{\begin{array}{l}
\frac{\pi}{2} \sin (n x), \quad \text { for } \quad|x| \leqslant 1, \\
0, \text { for } x \text { at the other points; } n=50 .
\end{array}\right.
$$

The simulation results with varied $B$ are:

\begin{tabular}{ccccc}
\hline$B$ & 10 & 20 & 50 & 100 \\
\hline$t_{50}^{*}$ & 0.5 & 0.5 & 0.48 & 0.49 \\
\hline$\widetilde{M}_{50}\left(t_{50}^{*}\right)$ & 20.885 & 20.885 & 20.891 & 20.972 \\
\hline
\end{tabular}

With the same kernel and $B=10$, the simulation results with varied $n$ are:

\begin{tabular}{ccccc}
\hline$n$ & 50 & 100 & 500 & 1000 \\
\hline$t_{n}^{*}$ & 0.5 & 0.5 & 0.5 & 0.5 \\
\hline$\widetilde{M}_{n}\left(t_{n}^{*}\right)$ & 20.885 & 20.783 & 20.326 & 20.529 \\
\hline
\end{tabular}

From the simulation above, we can draw the following conclusions:

(1) The estimates of jump positions and jump magnitudes derived from $\widetilde{M}_{n}(t)$ are also consistent. $\widetilde{M}_{n}(t)$ and $M_{n}(t)$ are derived from two different forms of kernel estimate of regression function. 
(2) The first kernel used in simulation does not satisfy Lip(1) condition. But the results are also satisfactory. So the Lip(1) condition of kernels can probably be cancelled.

(3) It is unnecessary for B to be very large. From theoretical analysis, $B \geqslant 1 / h_{n}$ is enough. The simulation results are coincided with it.

\section{References}

[1] QIU, P.: Estimation of a kind of jump regression functions, Chinese Journal of System Science and Mathematics, (To appear), (1990).

[2] Cheng, X. R.: Hypothesis testing and interval estimation of the model which has only one changepoint, Scientia Sinica 8A, (1988), 1-13.

[3] Cheng, K. F. and Lin, P. E.: Nonparametric estimation of a regression, Z. Wahrsch, Verw. Gebtete 57, (1981), 223-233.

Received October 4, 1990 